\title{
Sudakov representation of the Cachazo-He-Yuan scattering equations formalism
}

\author{
Grigorios Chachamis, ${ }^{a}$ Diego Medrano Jiménez, ${ }^{a}$ Agustín Sabio Vera ${ }^{a}$ \\ and Miguel Á. Vázquez-Mozo ${ }^{b}$ \\ ${ }^{a}$ Instituto de Física Teórica UAM/CSIC \& Universidad Autónoma de Madrid, \\ C/Nicolás Cabrera 15, E-28049 Madrid, Spain \\ ${ }^{b}$ Departamento de Física Fundamental, Universidad de Salamanca, \\ Plaza de la Merced s/n, E-37008 Salamanca, Spain \\ E-mail: chachamis@gmail.com, d.medrano@csic.es, \\ a.sabio.vera@gmail.com, Miguel.Vazquez-Mozo@cern.ch
}

ABSTRACT: We show that the use of Sudakov variables greatly simplifies the study of the solutions to the scattering equations in the Cachazo-He-Yuan formalism. We work in the center-of-mass frame for the two incoming particles, which partially fixes the $\operatorname{SL}(2, \mathbb{C})$ redundancy in the integrand defining the scattering amplitudes, the remaining freedom translates into a global shift in the azimuthal angle of the outgoing particles. Studying four- and five-particle amplitudes, we see how an appropriate choice of this phase allows for algebraic simplifications when finding solutions to the scattering equations, as well as in the expression of the scattering amplitudes in terms of the locations of the punctures in the sphere. These punctures themselves are remarkably simple functions of the Sudakov parameters.

KEYwords: Perturbative QCD, Scattering Amplitudes

ARXIV EPRINT: 1712.04288 


\section{Contents}

1 Introduction 1

2 The CHY formalism for scattering amplitudes 2

2.1 Momentum space and the punctured sphere 2

2.2 Scattering amplitudes 3

2.3 Fairlie's solution to the scattering equations 4

$\begin{array}{llr}3 & \text { Incoming momenta } & 6\end{array}$

4 Sudakov representation of the scattering equations: the four-point case 8

$\begin{array}{llr}4.1 & \text { Punctures on the Riemann sphere } & 9\end{array}$

$\begin{array}{ll}4.2 \text { The scattering equations and the four-point amplitude } & 11\end{array}$

5 Sudakov representation of the five-point amplitude $\quad 14$

$\begin{array}{lll}5.1 \text { Location of the punctures } & 14\end{array}$

$\begin{array}{lll}5.2 & \text { Scattering equations } & 17\end{array}$

$\begin{array}{lll}5.3 & \text { Scalar scattering amplitude } & 19\end{array}$

6 Conclusions and outlook $\quad 22$

\section{Introduction}

Scattering amplitudes are the building blocks for the computation of observables in quantum field theory and string theory (see [1] for a review). The progress in their understanding and calculation in recent years has been enormous. Usual Feynman diagram techniques become too complicated as the number of external legs or loops increases and any alternative procedures are always desirable. The Cachazo-He-Yuan (CHY) formalism $[2,3]$ represents a very promising route in this direction. It presents $n$-point amplitudes as an $(n-3)$-dimensional integral over the moduli space of $n$-punctured spheres, fully localized on the solutions to the so-called scattering equations (SE) [2, 4-7]. A proof of this formula for arbitrary $n$ was given by Dolan and Goddard [8]. The CHY proposal for the calculation of tree-level scattering amplitudes has an interpretation in terms of ambitwistor strings [913] defined on a Riemann surface at genus zero. At loop level, supergravity integrands of four-point amplitudes at one and two loops have been obtained when introducing higher genus $[11,14-16]$. Other connections to string theory amplitudes can be found in, for example, [17-19].

The main difficulty with the CHY strategy is that the number of integrals defining the $n$-point $S$-matrix elements, although being compensated by a delta function, grows very rapidly. The reason is that the number of integrations to be carried out goes like 
the number of solutions to the SE, which is $(n-3)$ ! for the $n$-point amplitude. There has been steady progress in the understanding of the solutions to the SE and the calculation of amplitudes obtained from them (see, for example, [20-27]).

In this work we focus on the physical interpretation of the solutions to the SE in terms of the positions of the associated punctures on the Riemann sphere. We find that Sudakov variables [28], which parametrize outgoing momenta in terms of its projections on two incoming momenta and a vector transverse to their collision axis, are a very efficient way to present the solutions to the SE, since they naturally encode momentum conservation. When evaluating the scattering amplitudes it is also useful to work in the center-of-mass frame of the two incoming particles. This is equivalent to partially fixing the $\operatorname{SL}(2, \mathbb{C})$ redundancy, localizing two of the punctures at opposite poles of the sphere while leaving a third puncture free. This residual symmetry corresponds to the freedom in the choice of the origin for the azimuthal angle with respect to the axis defined by the incoming particles. Choosing this global phase wisely allows for a simple representation of the scattering amplitude in terms of the position of the punctures on the sphere, which also admit a simple representation.

In section 2, we review those aspects of the $\mathrm{CHY}$ approach which are of special interest for our work. In particular, we discuss in detail the solution to the SE found in [6], which exists for any number of external particles in four dimensions and which we write in terms of the rapidities and the azimuthal angles of the emitted particles. In section 3, after identifying two of the particles participating in the scattering as incoming, we work in their center-of-mass frame taking the $z$ axis as their direction of flight. This is done through a double scaling limit involving the rapidities and transverse momenta.

Section 4 is devoted to describe the use of Sudakov variables in the simple case of fourparticle scattering. The punctures associated with the outgoing momenta are characterized by a single Sudakov variable and one azimuthal angle, which parametrizes circles on the Riemann sphere. Then we calculate the four-point amplitude for a scalar cubic theory using this representation. In section 5 we analyze the more complicated case of the fivepoint amplitudes. In this case four Sudakov variables and two azimuthal angles are needed to parametrize the system of SE and the punctures positions. We show how to obtain a second solution to the SE as the complex conjugate of the one previously discussed. At the end of this section we evaluate the corresponding amplitude for a scalar theory and express it as a simple function of the Sudakov variables. Finally, in section 6 we present our conclusions and directions for future work.

\section{The CHY formalism for scattering amplitudes}

\subsection{Momentum space and the punctured sphere}

In a scattering problem the data are codified in a set of $n$ on-shell $D$-dimensional momenta $p_{i}^{\mu}$ satisfying energy-momentum conservation

$$
\sum_{i=1}^{n} p_{i}=0,
$$

modulo Lorentz transformations. The departing point of the CHY formalism $[2,3]$ is a mapping from these momenta into an internal space on the $n$-punctured Riemann sphere 
parametrized by the variables $\sigma_{i} \in \mathbb{C P}^{1}$ (with $i=1, \ldots, n$ ) through the identity

$$
p_{j}^{\mu}=\oint_{\left|z-\sigma_{j}\right|=\epsilon} \frac{d z}{2 \pi i} \frac{v^{\mu}(z)}{\prod_{k=1}^{n}\left(z-\sigma_{k}\right)},
$$

where

$$
v^{\mu}(z)=\sum_{j=1}^{n} p_{j}^{\mu} \prod_{\substack{k=1 \\ k \neq j}}^{n}\left(z-\sigma_{k}\right)
$$

Due to momentum conservation, this is a vector-valued polynomial of degree $n-2$ satisfying $v(z)^{2}=0$. This latter null condition for $v(z)$ implies the SE

$$
\mathcal{S}_{i}(\sigma) \equiv \sum_{j \neq i}^{n} \frac{s_{i j}}{\sigma_{i j}}=0
$$

where $\sigma_{i j} \equiv \sigma_{i}-\sigma_{j}$ and we have introduced the Mandelstam invariants

$$
s_{i j}=\left(p_{i}+p_{j}\right)^{2}=2 p_{i} \cdot p_{j} .
$$

Although there are $n$ equations, only $n-3$ are linearly independent as a consequence of $\mathrm{SL}(2, \mathbb{C})$ invariance. These SE have a total of $(n-3)$ ! solutions mapping the space of kinematic invariants into $(n-3)$ ! points in the moduli space of $n$-punctured spheres.

The SE first appeared in ref. [7] in the study of the ground state configuration for the Koba-Nielsen representation of scattering amplitudes of open strings,

$$
A_{n}=\int d \sigma_{2} \ldots d \sigma_{n-2} \prod_{\substack{i, j=1 \\ i>j}}^{n-1} \sigma_{i j}^{-2 \alpha^{\prime} p_{i} \cdot p_{j}}
$$

where $0=\sigma_{1}<\sigma_{2}<\cdots<\sigma_{n-1}=1$. The dominant saddle-point region was investigated by Gross and Mende [4, 5] in the closed string case and by Gross and Mañes for open strings [29]. In both cases, all $s_{i j}$ are taken to be large simultaneously, corresponding precisely to eq. (2.4).

\section{$2.2 \quad$ Scattering amplitudes}

Remarkably, as shown in [2, 3], all tree-level $n$-point Yang-Mills amplitudes in $D$ dimensions can be obtained from the following integral representation with support on the solutions to the SE:

$$
\mathcal{A}_{n}=i g^{n-2} \int \frac{d^{n} \sigma}{\operatorname{Vol}[\operatorname{SL}(2, \mathbb{C})]} \sigma_{k l} \sigma_{l m} \sigma_{m k} \prod_{i \neq k, l, m} \delta\left(\sum_{j \neq i}^{n} \frac{2 p_{i} \cdot p_{j}}{\sigma_{i j}}\right) I_{L} I_{R} .
$$

The invariance of this expression under $\mathrm{SL}(2, \mathbb{C})$ transformations of the $\sigma_{i}$ guarantees that the result is independent of the choice of $\{k, l, m\}$. In the integrand, $I_{L}$ carries the color traces

$$
I_{L}=\sum_{\beta \in S_{n} / \mathbb{Z}_{n}} \frac{\operatorname{Tr}\left(T^{a_{\beta(1)}} T^{a_{\beta(2)}} \cdots T^{a_{\beta(n)}}\right)}{\sigma_{\beta(1) \beta(2)} \sigma_{\beta(2) \beta(3)} \cdots \sigma_{\beta(n) \beta(1)}},
$$


with the sum running over non-cyclic permutations. The second factor

$$
I_{R}=\operatorname{Pf}^{\prime} M_{n},
$$

is the reduced Pfaffian of the $2 n \times 2 n$ antisymmetric matrix

$$
M_{n}=\left(\begin{array}{cc}
M_{A} & -M_{C}^{T} \\
M_{C} & M_{B}
\end{array}\right) .
$$

where the block matrices $M_{A}, M_{B}$, and $M_{C}$ are given by

$$
\begin{aligned}
& M_{A}^{i j}=\left\{\begin{array}{ll}
\frac{p_{i} \cdot p_{j}}{\sigma_{i j}} & \text { for } i \neq j \\
0 & \text { for } i=j
\end{array},\right. \\
& M_{B}^{i j}=\left\{\begin{array}{ll}
\frac{\epsilon_{i} \cdot \epsilon_{j}}{\sigma_{i j}} & \text { for } i \neq j \\
0 & \text { for } i=j
\end{array},\right. \\
& M_{C}^{i j}= \begin{cases}\frac{\epsilon_{i} \cdot p_{j}}{\sigma_{i j}} & \text { for } i \neq j \\
-\sum_{k \neq i} \frac{\epsilon_{i} \cdot p_{k}}{\sigma_{i k}} & \text { for } i=j\end{cases}
\end{aligned}
$$

with $\epsilon_{i}$ the polarization vector of the $i$-th gauge boson. The reduced Pfaffian in eq. (2.9) is defined as the Pfaffian of the matrix whose entries are obtained from $M_{n}$ by removing the $k$-th row and $\ell$-th column and multiplied by $(-1)^{k+\ell} \sigma_{k \ell}^{-1}$.

Since the determinant of an antisymmetric matrix is the square of its Pfaffian, it is natural that graviton amplitudes in Einstein-Hilbert gravity can also be written in the form (2.7), with the gauge theory factor $I_{L}$ shown in eq. (2.8) replaced by a second copy of the reduced Pfaffian

$$
\begin{aligned}
& I_{L}=\operatorname{Pf}^{\prime} M_{n}, \\
& I_{R}=\operatorname{Pf}^{\prime} M_{n} .
\end{aligned}
$$

This exhibits the double-copy structure of graviton amplitudes, already found in many other physical setups [30-35]. Similarly, biadjoint scalar amplitudes can be obtained from eq. (2.7) substituting the reduced Pfaffian in $I_{R}$ by a second copy of the gauge theory factor (2.8), thus implementing the zeroth copy prescription [36].

\subsection{Fairlie's solution to the scattering equations}

In this section we discuss a definite solution to the SE discussed by Fairlie in [6] (see also [7]), which always exists for any multiplicity $n$. It has the form

$$
\begin{aligned}
\sigma_{j} & =\frac{p_{j}^{0}+p_{j}^{3}}{p_{j}^{1}-i p_{j}^{2}}=\frac{\left(p_{j}^{0}+p_{j}^{3}\right)\left(p_{j}^{1}+i p_{j}^{2}\right)}{\left(p_{j}^{1}\right)^{2}+\left(p_{j}^{2}\right)^{2}} \\
& =\frac{\left(p_{j}^{0}+p_{j}^{3}\right)\left(p_{j}^{1}+i p_{j}^{2}\right)}{\left(p_{j}^{0}\right)^{2}-\left(p_{j}^{3}\right)^{2}}=\frac{p_{j}^{1}+i p_{j}^{2}}{p_{j}^{0}-p_{j}^{3}},
\end{aligned}
$$


where we work in $D=4$ with the mostly-minus signature. Since $\sigma_{i}$ admits two expressions in terms of the momentum components, we can write two alternative identities to be satisfied by the differences $\sigma_{i j}$

$$
\begin{aligned}
& \sigma_{i j}\left(p_{i}^{1}-i p_{i}^{2}\right)\left(p_{j}^{0}-p_{j}^{3}\right)=p_{i} \cdot p_{j}-p_{i}^{0} p_{j}^{3}+p_{i}^{3} p_{j}^{0}-i p_{i}^{1} p_{j}^{2}+i p_{i}^{2} p_{j}^{1}, \\
& \sigma_{i j}\left(p_{j}^{1}-i p_{j}^{2}\right)\left(p_{i}^{0}-p_{i}^{3}\right)=-p_{i} \cdot p_{j}+p_{j}^{0} p_{i}^{3}-p_{j}^{3} p_{i}^{0}+i p_{j}^{1} p_{i}^{2}-i p_{j}^{2} p_{i}^{1} .
\end{aligned}
$$

Subtracting both equations, we arrive at the expression

$$
\left(p_{i}^{1}-i p_{i}^{2}\right)\left(p_{j}^{0}-p_{j}^{3}\right)-\left(p_{j}^{1}-i p_{j}^{2}\right)\left(p_{i}^{0}-p_{i}^{3}\right)=2 \frac{p_{i} \cdot p_{j}}{\sigma_{i j}} .
$$

We can use this identity to explicitly check that (2.13) is indeed a solution to the SE. Summing over $j$ with $j \neq i$ we have

$$
\begin{aligned}
2 \sum_{\substack{j=1 \\
j \neq i}}^{n} \frac{p_{i} \cdot p_{j}}{\sigma_{i j}} & =\left(p_{i}^{1}-i p_{i}^{2}\right) \sum_{\substack{j=1 \\
j \neq i}}^{n}\left(p_{j}^{0}-p_{j}^{3}\right)-\left(p_{i}^{0}-p_{i}^{3}\right) \sum_{\substack{j=1 \\
j \neq i}}^{n}\left(p_{j}^{1}-i p_{j}^{2}\right) \\
& =-\left(p_{i}^{1}-i p_{i}^{2}\right)\left(p_{i}^{0}-p_{i}^{3}\right)+\left(p_{i}^{0}-p_{i}^{3}\right)\left(p_{i}^{1}-i p_{i}^{2}\right)=0,
\end{aligned}
$$

where we have made use of momentum conservation.

It is possible to bring these solutions into a more physical representation if we use the following parametrization of on-shell momenta $p_{j}$

$$
p_{j}=p_{j}^{\perp}\left(\cosh Y_{j}, \cos \phi_{j}, \sin \phi_{j}, \sinh Y_{j}\right),
$$

where $Y_{j}$ is the rapidity, $\phi_{j}$ the azimuthal angle, and the overall scale $p_{j}^{\perp}$ equals the modulus of the transverse component of the momentum. To connect this representation with the one in terms of the $n$-punctured sphere, we notice that $p_{j}$ can be alternatively written as

$$
p_{j}=\omega_{j}\left(1, \mathbf{u}_{j}\right),
$$

where we have introduced the unit vector

$$
\mathbf{u}_{j}=\left(x_{j}, y_{j}, z_{j}\right), \quad \mathbf{u}_{j}^{2}=1,
$$

and $\omega_{j}$ is the energy of the $j$-th particle. Using this parametrization it is glaring how a null momentum is completely specified by the energy of the particle and its direction of flight, corresponding to a point on $\mathbb{R} \times \mathbb{S}^{2}$. Points on the celestial sphere $\mathbb{S}^{2}$ can be parametrized either using stereographic coordinates $\zeta_{j}$ or the polar and azimuthal angles $\left(\theta_{j}, \phi_{j}\right)$. They are related by the following identities

$$
\begin{aligned}
& x_{j}=\sin \theta_{j} \cos \phi_{j}=\frac{2 e^{Y_{j}} \cos \phi_{j}}{1+e^{2 Y_{j}}}=\frac{\zeta_{j}+\bar{\zeta}_{j}}{1+\bar{\zeta}_{j} \bar{\zeta}_{j}}, \\
& y_{j}=\sin \theta_{j} \sin \phi_{j}=\frac{2 e^{Y_{j}} \sin \phi_{j}}{1+e^{2 Y_{j}}}=i \frac{\bar{\zeta}_{j}-\zeta_{j}}{1+\zeta_{j} \bar{\zeta}_{j}}, \\
& z_{j}=\cos \theta_{j}=\frac{e^{2 Y_{j}}-1}{1+e^{2 Y_{j}}}=\frac{\zeta_{j} \bar{\zeta}_{j}-1}{1+\zeta_{j} \bar{\zeta}_{j}},
\end{aligned}
$$




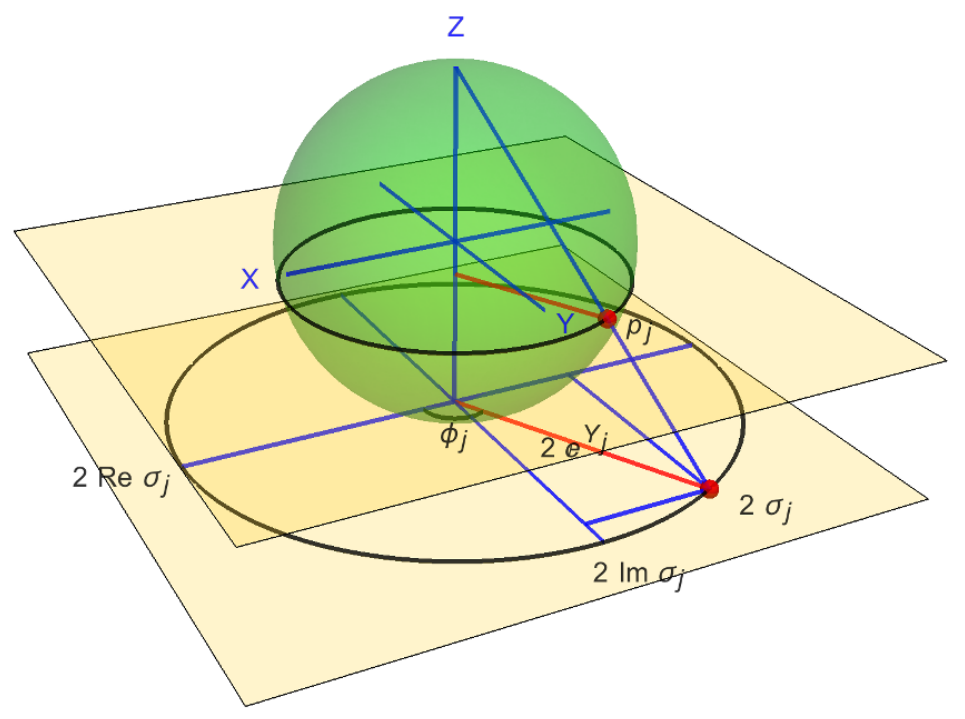

Figure 1. Geometric interpretation of the rapidity $Y_{j}$ and azimuthal angle $\phi_{j}$.

which can be inverted to give

$$
\begin{aligned}
& \zeta_{j}=e^{Y_{j}} e^{i \phi_{j}}=\frac{\sin \theta_{j}}{1-\cos \theta_{j}} e^{i \phi_{j}}=\cot \frac{\theta_{j}}{2} e^{i \phi_{j}}, \\
& \bar{\zeta}_{j}=e^{Y_{j}} e^{-i \phi_{j}}=\frac{\sin \theta_{j}}{1-\cos \theta_{j}} e^{-i \phi_{j}}=\cot \frac{\theta_{j}}{2} e^{-i \phi_{j}} .
\end{aligned}
$$

This leads to the following parametrization of the particle momenta in terms of its energy and the stereographic coordinates on $\mathbb{S}^{2}$

$$
p_{j}=\omega_{j}\left(1, \frac{\zeta_{j}+\bar{\zeta}_{j}}{1+\zeta_{j} \bar{\zeta}_{j}}, i \frac{\bar{\zeta}_{j}-\zeta_{j}}{1+\zeta_{j} \bar{\zeta}_{j}}, \frac{\zeta_{j} \bar{\zeta}_{j}-1}{1+\zeta_{j} \bar{\zeta}_{j}}\right)
$$

Using the previous representation of the particle momenta, we see that Fairlie's solution (2.13) to the SE is simply given by

$$
\sigma_{j}=\zeta_{j}=e^{Y_{j}+i \phi_{j}}
$$

Since we will make frequent use of this representation in the following, some remarks are in order. In figure 1 we have represented a point in the celestial sphere and its image on the complex plane whose origin coincides with the south pole. The direction of flight of a particle with momentum $p_{j}$ labelled by the complex coordinate $\zeta_{j}$ is mapped onto the point $2 \sigma_{j}$ on that plane. At fixed rapidity $Y_{j}$, the points lie on a circumference of radius $2 e^{Y_{j}}$ parametrized by the azimuthal angle $\phi_{j}$.

\section{Incoming momenta}

In this section we investigate the structure on the punctured sphere for the two incoming particles with momenta $p$ and $q$ in a general process in which the particles in the final 
state have momenta $p_{i}$ (with $i=1, \ldots, n-2$ ). We will consider the case when the two incoming particles' spatial momenta lie along the $z$ axis. It is convenient to work first with the parametrization in terms of rapidities and azimuthal angles introduced in eq. (2.17)

$$
\begin{aligned}
& p=\ell\left(\cosh Y_{p}, \cos \phi, \sin \phi, \sinh Y_{p}\right), \\
& q=\ell\left(\cosh Y_{q},-\cos \phi,-\sin \phi, \sinh Y_{q}\right),
\end{aligned}
$$

where we have set both transverse momenta equal, $p^{\perp}=q^{\perp} \equiv \ell$. To study the limit of vanishing transverse momenta, we take $\ell \rightarrow 0$ and $\left|Y_{p}\right|,\left|Y_{q}\right| \rightarrow \infty$, while keeping the center-of-mass energy

$$
s=2 p \cdot q=2 \ell^{2}\left[1+\cosh \left(Y_{p}-Y_{q}\right)\right]
$$

finite. This limit can be implemented by introducing a parameter $\epsilon$

$$
Y_{p}=-Y_{q}=-\log \epsilon,
$$

that we eventually take to zero. A look at eq. (3.2) shows that in order to keep $s$ finite we are forced to take the double scaling limit

$$
\epsilon \longrightarrow 0, \quad \ell \longrightarrow 0 \quad \text { with } \quad \frac{\ell}{\epsilon}=\sqrt{s}
$$

in which the incoming momenta take the form

$$
\begin{aligned}
& p \longrightarrow \frac{\sqrt{s}}{2}(1,0,0,1), \\
& q \longrightarrow \frac{\sqrt{s}}{2}(1,0,0,-1) .
\end{aligned}
$$

We can rephrase this double scaling in terms of the position of the corresponding punctures on the sphere $\left\{\sigma_{p}, \sigma_{q}\right\}$, which satisfy the identity

$$
\frac{\sigma_{p}}{\sigma_{q}}+\frac{\sigma_{q}}{\sigma_{p}}=2-\frac{s}{\ell^{2}} .
$$

Equation (2.23) shows that for small $\epsilon$ the two punctures are located on a small circle around the north and south poles of the Riemann sphere, which shrinks to a point when $\epsilon \rightarrow 0$, namely

$$
\begin{aligned}
\sigma_{p} & =e^{Y_{p}+i \phi}=\frac{e^{i \phi}}{\epsilon} \longrightarrow \infty, \\
\sigma_{q} & =-e^{Y_{q}+i \phi}=-\epsilon e^{i \phi} \longrightarrow 0 .
\end{aligned}
$$

These punctures can be alternatively labelled by the unit vectors $\mathbf{u}_{p}$ and $\mathbf{u}_{q}$ defined by eq. (2.18). In our case, they take the form

$$
\begin{aligned}
& \mathbf{u}_{p}=\left(\frac{\cos \phi}{\cosh Y_{p}}, \frac{\sin \phi}{\cosh Y_{p}}, \tanh Y_{p}\right), \\
& \mathbf{u}_{q}=\left(-\frac{\cos \phi}{\cosh Y_{q}},-\frac{\sin \phi}{\cosh Y_{q}}, \tanh Y_{q}\right),
\end{aligned}
$$




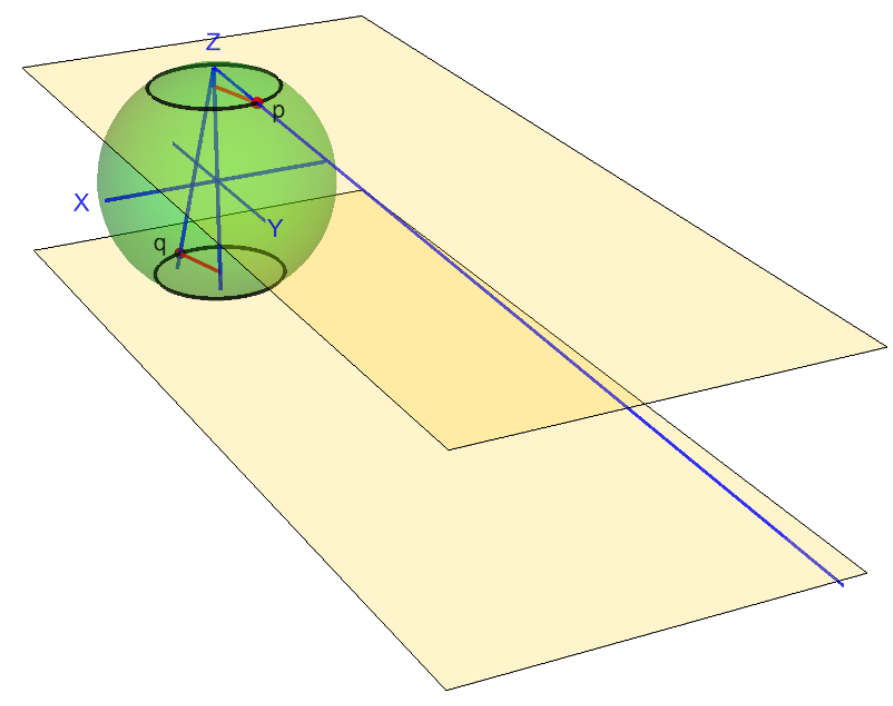

Figure 2. Stereographic projection for two incoming particles whose momenta lie close to the $z$ axis.

whose projections onto the equatorial plane lie on circles with respective radii

$$
\begin{aligned}
& R_{p}=\frac{1}{\cosh Y_{p}}, \\
& R_{q}=\frac{1}{\cosh Y_{q}},
\end{aligned}
$$

which shrink to zero as $\left|Y_{p, q}\right| \rightarrow \infty$ (i.e., $\epsilon \rightarrow 0$ ).

The geometric setup for the configuration discussed here is illustrated in figure 2 , where we show the punctures associated with the incoming particles very close to the north and south poles of the Riemann sphere. The value of $\phi$ is ambiguous for points on the $z$ axis and without loss of generality we can set it to zero from now on, since this angle is a mere artefact of the way we take the limit. On the complex plane this means that the limits $\sigma_{q} \rightarrow 0$ and $\sigma_{p} \rightarrow \infty$ are taken along the real axis.

\section{Sudakov representation of the scattering equations: the four-point case}

After introducing our setup and conventions, we turn to study the formulation of the SE formalism in terms of Sudakov parameters. We begin with the simplest case, that of a general four-point scattering amplitude with incoming and outgoing momenta respectively given by $p, q$ and $p^{\prime}, q^{\prime}$, which are constrained by momentum conservation

$$
p+q-p^{\prime}-q^{\prime}=0
$$

We parametrize the two incoming momenta $p$ and $q$ as explained in the previous section. 


\subsection{Punctures on the Riemann sphere}

In the CHY formalism $[2,3]$, the momenta $\left\{p, q, p^{\prime}, q^{\prime}\right\}$ are mapped into the moduli space of spheres with four punctures, located respectively at the points $\left\{\sigma_{p}, \sigma_{q}, \sigma_{p^{\prime}}, \sigma_{q^{\prime}}\right\} \in \mathbb{C P}^{1}$. This is implemented by the identities

$$
\begin{aligned}
p^{\mu} & =\oint_{\left|z-\sigma_{p}\right|=\epsilon} \frac{d z}{2 \pi i} \omega^{\mu}(z), \\
q^{\mu} & =\oint_{\left|z-\sigma_{q}\right|=\epsilon} \frac{d z}{2 \pi i} \omega^{\mu}(z), \\
p^{\prime \mu} & =-\oint_{\left|z-\sigma_{p^{\prime}}\right|=\epsilon} \frac{d z}{2 \pi i} \omega^{\mu}(z), \\
q^{\prime \mu} & =-\oint_{\left|z-\sigma_{q^{\prime}}\right|=\epsilon} \frac{d z}{2 \pi i} \omega^{\mu}(z),
\end{aligned}
$$

where the meromorphic function $\omega^{\mu}(z)$ is fully determined by the condition that it has poles at the location of the punctures whose residues are the corresponding particle momenta

$$
\omega^{\mu}(z)=\frac{p^{\mu}}{z-\sigma_{p}}+\frac{q^{\mu}}{z-\sigma_{q}}-\frac{p^{\prime \mu}}{z-\sigma_{p^{\prime}}}-\frac{q^{\prime \mu}}{z-\sigma_{q^{\prime}}} .
$$

The incoming momenta are parametrized as shown in eq. (3.1) with $\phi=0$. For the outgoing particles, on the other hand, we write their momenta introducing a Sudakov [28] representation. Due to momentum conservation (4.1), it is enough to parametrize the combination

$$
q_{1} \equiv p-p^{\prime}=\alpha p+\beta q+\mathbf{q}_{1}
$$

with

$$
\mathbf{q}_{1}=q_{1}^{\perp}\left(0, \cos \theta_{1}, \sin \theta_{1}, 0\right) .
$$

Then, the momentum $p^{\prime}$ can be written as

$$
\begin{aligned}
p^{\prime}=p-q_{1}= & \ell\left((1-\alpha) \cosh Y_{p}-\beta \cosh Y_{q}, 0,0,(1-\alpha) \sinh Y_{p}-\beta \sinh Y_{q}\right) \\
& +\left(0,(1-\alpha+\beta) \ell-q_{1}^{\perp} \cos \theta_{1},-q_{1}^{\perp} \sin \theta_{1}, 0\right) \\
\longrightarrow & \left(\frac{\sqrt{s}}{2}(1-\alpha-\beta),-q_{1}^{\perp} \cos \theta_{1},-q_{1}^{\perp} \sin \theta_{1}, \frac{\sqrt{s}}{2}(1-\alpha+\beta)\right),
\end{aligned}
$$

where in the last expression we have taken the double scaling limit (3.4). From this we read the particle energy

$$
\omega_{p^{\prime}}=\frac{\sqrt{s}}{2}(1-\alpha-\beta),
$$

whereas the on-shell condition leads to

$$
0=p^{\prime 2}=-s(1-\alpha) \beta-\left(q_{1}^{\perp}\right)^{2} \quad \Longrightarrow \quad\left|Q_{1}\right|^{2} \equiv\left(q_{1}^{\perp}\right)^{2}=s(\alpha-1) \beta,
$$


where we have introduced the notation

$$
Q_{j}=q_{j}^{\perp} e^{i \theta_{j}} .
$$

We repeat the same calculation for the momentum $q^{\prime}$ of the second outgoing particle. In terms of the Sudakov parameters, it reads

$$
\begin{aligned}
q^{\prime}=q+q_{1}= & \ell\left(\alpha \cosh Y_{p}+(1+\beta) \cosh Y_{q}, 0,0, \alpha \sinh Y_{p}+(1+\beta) \sinh Y_{q}\right) \\
& +\left(0,(\alpha-1-\beta) \ell+q_{1}^{\perp} \cos \theta_{1}, q_{1}^{\perp} \sin \theta_{1}, 0\right) \\
\longrightarrow & \left(\frac{\sqrt{s}}{2}(1+\alpha+\beta), q_{1}^{\perp} \cos \theta_{1}, q_{1}^{\perp} \sin \theta_{1}, \frac{\sqrt{s}}{2}(-1+\alpha-\beta)\right),
\end{aligned}
$$

where we have reabsorbed a sign in a shift of $\theta_{1}$ by $\pi$. Comparing with the expression for $p^{\prime}$ in eq. (4.6) we see that this reflects the fact that, in the center-of-mass frame, the two outgoing particles fly in opposite directions and therefore their azimuthal angles differ by $\pi$. The energy of the particle is given by

$$
\omega_{q^{\prime}}=\frac{\sqrt{s}}{2}(1+\alpha+\beta),
$$

whereas the on-shell condition $q^{\prime 2}=0$ leads to the constraint

$$
0=q^{\prime 2}=s \alpha(1+\beta)-\left(q_{1}^{\perp}\right)^{2} \quad \Longrightarrow \quad\left|Q_{1}\right|^{2} \equiv\left(q_{1}^{\perp}\right)^{2}=s \alpha(1+\beta) .
$$

Consistency with the value of $\left|Q_{1}\right|^{2}$ found from the on-shell condition $p^{2}=0$ in eq. (4.8) implies that $\alpha$ and $\beta$ are not independent, but rather satisfy

$$
\alpha+\beta=0 .
$$

This condition implies that

$$
\omega_{p^{\prime}}=\omega_{q^{\prime}}=\frac{\sqrt{s}}{2},
$$

as it behoves a four particle scattering in the center-of-mass frame.

Let us recall that for the four-point function, the SE only have one solution. Thus, it is enough to consider Fairlie's solution (2.13) reviewed in section 2.3. This being the case, the complex coordinate of the puncture in the sphere associated with the momentum $p^{\prime}$ is given by

$$
\sigma_{p^{\prime}} \equiv e^{Y_{p^{\prime}}+i \phi_{p^{\prime}}}=\frac{Q_{1}}{\beta \sqrt{s}}=\sqrt{\frac{1-\alpha}{\alpha}} e^{i\left(\theta_{1}+\pi\right)},
$$

where in using (4.8) to write the result in terms of $Q_{1}$ we have made a choice of phase for the square root. In addition, the projection of the associated unit vector $\mathbf{u}_{p^{\prime}}$

$$
\mathbf{u}_{p^{\prime}}=\frac{2}{\sqrt{s}}\left(q_{1}^{\perp} \cos \left(\theta_{1}+\pi\right), q_{1}^{\perp} \sin \left(\theta_{1}+\pi\right), \frac{\sqrt{s}}{2}(1-2 \alpha)\right),
$$


onto the equatorial plane lies on a circumference with radius

$$
R_{p^{\prime}}=2 \sqrt{\alpha(1-\alpha)}
$$

where we have used the on-shell condition (4.8). Going to the Riemann sphere representation, the complex coordinate of the puncture associated with the particle of momentum $q^{\prime}$ is

$$
\sigma_{q^{\prime}} \equiv e^{Y_{q^{\prime}}+i \phi_{q^{\prime}}}=\frac{Q_{1}}{(1-\alpha) \sqrt{s}}=\sqrt{\frac{\alpha}{1-\alpha}} e^{i \theta_{1}},
$$

where our choice of phase is consistent with the one used for $\sigma_{p^{\prime}}$ in eq. (4.15). Thus, we conclude

$$
\sigma_{q^{\prime}}=-\frac{1}{\sigma_{p^{\prime}}^{*}}=\sqrt{\frac{\alpha}{1-\alpha}} e^{i \theta_{1}}
$$

indicating that the two punctures are located on antipodal points on the sphere. This becomes obvious when computing the components of the unit vector $\mathbf{u}_{q^{\prime}}$

$$
\mathbf{u}_{q^{\prime}}=\frac{2}{\sqrt{s}}\left(q_{1}^{\perp} \cos \theta_{1}, q_{1}^{\perp} \sin \theta_{1},-\frac{\sqrt{s}}{2}(1-2 \alpha)\right) .
$$

Now, since after imposing (4.13) we see that $\mathbf{u}_{q^{\prime}}=-\mathbf{u}_{p^{\prime}}$, the projection of both vectors on the equatorial plane defines the same loci, namely a circumference with radius [cf. eq. (4.17)]

$$
R_{p^{\prime}}=R_{q^{\prime}}=2 \sqrt{\alpha(1-\alpha)}
$$

whereas their components along the direction of the incoming particles are

$$
Z_{p^{\prime}}=-Z_{q^{\prime}}=1-2 \alpha .
$$

In figure 3 we provide a pictorial example of the parametrization proposed above. The boundary of the moduli space of the sphere with four punctures is approached in the limits $\alpha \rightarrow 1$ or $\alpha \rightarrow 0$. They correspond to the coincidence limit in which the punctures associated with the outgoing particles collide with those of the incoming ones, located at the north and south pole of the Riemann sphere. In the case $\alpha=\frac{1}{2}$ the radii $R_{p^{\prime}}=R_{q^{\prime}}=2 \sqrt{\alpha(1-\alpha)}$ reach the maximum value and the outgoing particles are emitted along the equatorial plane.

\subsection{The scattering equations and the four-point amplitude}

In order to write the SE using the Sudakov parametrization, we need to compute the Mandelstam invariants (2.5) where, according to our conventions $p_{1}=p, p_{2}=q, p_{3}=-p^{\prime}$, and $p_{4}=-q^{\prime}$. For four particle scattering, they have the following explicit form

$$
\begin{aligned}
s_{p q} & =s_{p^{\prime} q^{\prime}}=s, \\
s_{p p^{\prime}} & =s_{q q^{\prime}}=-q_{1}^{2}=-t=s \alpha, \\
s_{p q^{\prime}} & =s_{q p^{\prime}}=-u=s(1-\alpha) .
\end{aligned}
$$




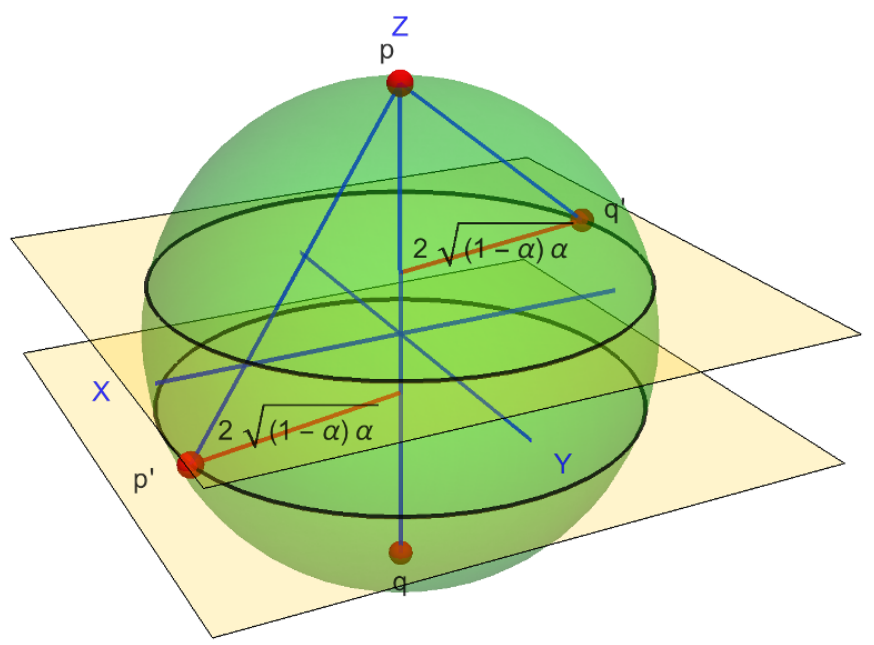

Figure 3. Punctures on the Riemann sphere for the four particle scattering with momenta $p+q \longrightarrow$ $p^{\prime}+q^{\prime}$. In the limits $\alpha \rightarrow 0,1$ the outgoing punctures collide with the incoming ones located at the poles.

Since $s$ is the only dimensionful quantity available, we use rescaled variables

$$
\begin{aligned}
s_{i j} & =s \widehat{s}_{i j}, \\
Q_{i} & =\sqrt{s} \widehat{Q}_{i} .
\end{aligned}
$$

It is straightforward to check that the SE associated with $p$ is trivially satisfied

$$
\frac{\mathcal{S}_{p}}{s}=\frac{\widehat{s}_{p q}}{\sigma_{p q}}-\frac{\widehat{s}_{p p^{\prime}}}{\sigma_{p p^{\prime}}}-\frac{\widehat{s}_{p q^{\prime}}}{\sigma_{p q^{\prime}}}=0
$$

since we have $\sigma_{p}=\infty$. In the case of the SE associated to $q$

$$
\frac{\mathcal{S}_{q}}{s}=\frac{\widehat{s}_{p q}}{\sigma_{q p}}-\frac{\widehat{s}_{q q^{\prime}}}{\sigma_{q q^{\prime}}}-\frac{\widehat{s}_{q p^{\prime}}}{\sigma_{q p^{\prime}}},
$$

we have a nontrivial cancellation. The first term vanishes again because $\sigma_{p}=\infty$ and we can use the explicit expressions

$$
\begin{gathered}
\sigma_{p^{\prime}}=-\frac{\widehat{Q}_{1}}{\alpha}, \\
\sigma_{q^{\prime}}=\frac{\widehat{Q}_{1}}{1-\alpha},
\end{gathered}
$$

together with $\sigma_{q}=0$. Using this Sudakov representation, it is easy to check that the SE is fulfilled

$$
\frac{\mathcal{S}_{q}}{s}=\frac{\widehat{s}_{q q^{\prime}}}{\sigma_{q^{\prime}}}+\frac{\widehat{s}_{q p^{\prime}}}{\sigma_{p^{\prime}}}=-\frac{\alpha(1-\alpha)}{\widehat{Q}_{1}}+\frac{(1-\alpha) \alpha}{\widehat{Q}_{1}}=0,
$$

and similarly for the two remaining $\mathrm{SE}$

$$
\begin{gathered}
\frac{\mathcal{S}_{p^{\prime}}}{s}=\frac{\widehat{s}_{p^{\prime} q}}{\sigma_{p^{\prime}}}-\frac{\widehat{s}_{p^{\prime} q^{\prime}}}{\sigma_{p^{\prime} q^{\prime}}}=-\frac{\alpha(1-\alpha)}{\widehat{Q}_{1}}+\frac{\alpha(1-\alpha)}{\widehat{Q}_{1}}=0, \\
\frac{\mathcal{S}_{q^{\prime}}}{s}=\frac{\widehat{s}_{q q^{\prime}}}{\sigma_{q^{\prime}}}-\frac{\widehat{s}_{p^{\prime} q^{\prime}}}{\sigma_{q^{\prime} p^{\prime}}}=-\frac{\alpha(1-\alpha)}{\widehat{Q}_{1}}+\frac{\alpha(1-\alpha)}{\widehat{Q}_{1}}=0 .
\end{gathered}
$$


The Sudakov representation provides a very convenient framework for the evaluation of scattering amplitudes in the CHY formalism, notably simplifying the computations. To illustrate this, we focus now on the calculation of the four-point amplitude in a $\varphi^{3}$ scalar theory. According to the general prescription given in $[2,3]$, the amplitude can be written as the following integral supported on the solution to the SE

$$
\begin{aligned}
\mathcal{A}_{4}^{\varphi^{3}} & =\int d z_{p^{\prime}} \delta\left(\mathcal{S}_{p^{\prime}}\right) \frac{z_{p q}^{2} z_{q q^{\prime}}^{2} z_{q^{\prime} p}^{2}}{\left(z_{p q} z_{q q^{\prime}} z_{q^{\prime} p^{\prime}} z_{p^{\prime} p}\right)^{2}} \\
& =\int \frac{d z_{p^{\prime}}}{\left(z_{p^{\prime}}-\sigma_{q^{\prime}}\right)^{2}} \delta\left(\frac{s_{p^{\prime} q}}{z_{p^{\prime}}}-\frac{s_{p^{\prime} q^{\prime}}}{z_{p^{\prime}}-\sigma_{q^{\prime}}}\right),
\end{aligned}
$$

where all gauge generators are taken to be equal to one. Here we have partially fixed the $\mathrm{SL}(2, \mathbb{C})$ invariance by setting $z_{p}=\infty$ and $z_{q}=0$ while leaving the third one

$$
z_{q^{\prime}}=\sigma_{q^{\prime}}=\frac{Q_{1}}{(1-\alpha) \sqrt{s}}
$$

free. The integral defining the scattering amplitude has just one integration left over the position of the $p^{\prime}$ puncture. To carry out this integral, we notice that the argument of the delta function has a single root located at [see (4.29)]

$$
z_{p^{\prime}}=-\frac{Q_{1}}{\alpha \sqrt{s}}
$$

Evaluating the derivative of $S_{p^{\prime}}$ with respect to the integration variable at the zero (4.34) gives

$$
\left.\mathcal{J} \equiv \frac{\partial S_{p^{\prime}}}{\partial z_{p^{\prime}}}\right|_{z_{p^{\prime}}=-\frac{Q_{1}}{\alpha \sqrt{s}}}=\frac{s^{2} \alpha^{2}(\alpha-1)}{Q_{1}^{2}}+\frac{s \alpha^{2}(1-\alpha)^{2}}{Q_{1}^{2}}=\frac{s^{2} \alpha^{3}(\alpha-1)}{Q_{1}^{2}}
$$

so we can simply write

$$
\begin{aligned}
\mathcal{A}_{4}^{\varphi^{3}} & =\int d z_{p^{\prime}}\left[z_{p^{\prime}}-\frac{Q_{1}}{(1-\alpha) \sqrt{s}}\right]^{-2} \frac{Q_{1}^{2}}{s^{2} \alpha^{3}(\alpha-1)} \delta\left(z_{p^{\prime}}+\frac{Q_{1}}{\alpha \sqrt{s}}\right) \\
& =\left[\frac{s \alpha^{2}(1-\alpha)^{2}}{Q_{1}^{2}}\right]\left[\frac{Q_{1}^{2}}{s^{2} \alpha^{3}(\alpha-1)}\right]=\frac{(\alpha-1)}{s \alpha}=\frac{1}{s}+\frac{1}{t} .
\end{aligned}
$$

Notice that the phase introduced in $Q_{1}$, which contains the azimuthal angle dependence, cancels out in the final expression for the amplitude. This is only natural, since $\theta_{1}$ can be set to zero by using the residual $\mathrm{SL}(2, \mathbb{C})$ transformations leaving invariant the position of the punctures associated with the incoming particles. Using this Sudakov parametrization, we see how the boundary of the 4-punctured sphere corresponding to the limit $\alpha \rightarrow 0$ is dominated by the $t=0$ pole, while at the other branch of the boundary $\alpha \rightarrow 1$ the amplitude vanishes. At the equator $\alpha=\frac{1}{2}$ the amplitude is completely dominated by the pole at $s=0$. 


\section{Sudakov representation of the five-point amplitude}

After the analysis of the four-point amplitude, we turn to the scattering of five particles which enjoys some more interesting features, mainly the existence of a second solution to the SE besides Fairlie's. To fix notation, we will now study a generic five-point scattering amplitude of particles with momenta $p+q \rightarrow p^{\prime}+k+q^{\prime}$ satisfying the momentum conservation identity

$$
p+q-p^{\prime}-k-q^{\prime}=0 .
$$

\subsection{Location of the punctures}

The mapping between particle momenta and the puncture positions is provided by the relations listed in eq. (4.2) supplemented with the one for $k$

$$
k^{\mu}=-\oint_{\left|z-\sigma_{k}\right|=\epsilon} \frac{d z}{2 \pi i} \omega^{\mu}(z),
$$

where now the meromorphic function $\omega^{\mu}(z)$ is given by

$$
\omega^{\mu}(z)=\frac{p^{\mu}}{z-\sigma_{p}}+\frac{q^{\mu}}{z-\sigma_{q}}-\frac{p^{\prime \mu}}{z-\sigma_{p^{\prime}}}-\frac{k^{\mu}}{z-\sigma_{k}}-\frac{q^{\prime \mu}}{z-\sigma_{q^{\prime}}} .
$$

To parametrize the momenta, we introduce two pairs of Sudakov parameters $\left\{\alpha_{1}, \beta_{1}\right\}$ and $\left\{\alpha_{2}, \beta_{2}\right\}$ such that

$$
\begin{aligned}
q_{1} & =p-p^{\prime}=\alpha_{1} p+\beta_{1} q+\mathbf{q}_{1}, \\
q_{2} & =q^{\prime}-q=\alpha_{2} p+\beta_{2} q+\mathbf{q}_{2}, \\
k & =q_{1}-q_{2}=\left(\alpha_{1}-\alpha_{2}\right) p+\left(\beta_{1}-\beta_{2}\right) q+\mathbf{q}_{1}-\mathbf{q}_{2},
\end{aligned}
$$

where the transverse vectors have components

$$
\mathbf{q}_{i}=q_{i}^{\perp}\left(0, \cos \theta_{i}, \sin \theta_{i}, 0\right) .
$$

Using again the notation introduced in eq. (4.9), and taking the double scaling limit (3.4), we have

$$
\begin{aligned}
p^{\prime}=p-q_{1}= & \ell\left(\left(1-\alpha_{1}\right) \cosh Y_{p}-\beta_{1} \cosh Y_{q}, 0,0,\left(1-\alpha_{1}\right) \sinh Y_{p}-\beta_{1} \sinh Y_{q}\right) \\
& +\left(0,\left(1-\alpha_{1}+\beta_{1}\right) \ell-q_{1}^{\perp} \cos \theta_{1},-q_{1}^{\perp} \sin \theta_{1}, 0\right) \\
\longrightarrow & \left(\frac{\sqrt{s}}{2}\left(1-\alpha_{1}-\beta_{1}\right),-q_{1}^{\perp} \cos \theta_{1},-q_{1}^{\perp} \sin \theta_{1}, \frac{\sqrt{s}}{2}\left(1-\alpha_{1}+\beta_{1}\right)\right) .
\end{aligned}
$$

A similar analysis can be repeated for the remaining two outgoing particles. In terms of the Sudakov parameters, their momenta take the form

$$
\begin{aligned}
q^{\prime}=q+q_{2}= & \ell\left(\alpha_{2} \cosh Y_{p}+\left(1+\beta_{2}\right) \cosh Y_{q}, 0,0, \alpha_{2} \sinh Y_{p}+\left(1+\beta_{2}\right) \sinh Y_{q}\right) \\
& +\left(0,\left(\alpha_{2}-\beta_{2}-1\right) \ell+q_{2}^{\perp} \cos \theta_{2}, q_{2}^{\perp} \sin \theta_{2}, 0\right) \\
\longrightarrow & \left(\frac{\sqrt{s}}{2}\left(1+\alpha_{2}+\beta_{2}\right), q_{2}^{\perp} \cos \theta_{2}, q_{2}^{\perp} \sin \theta_{2}, \frac{\sqrt{2}}{2}\left(-1+\alpha_{2}-\beta_{2}\right)\right),
\end{aligned}
$$




$$
\begin{aligned}
& k=q_{1}-q_{2}= \ell\left(\left(\alpha_{1}-\alpha_{2}\right) \cosh Y_{p}+\left(\beta_{1}-\beta_{2}\right) \cosh Y_{q}, 0\right. \\
&\left.0,\left(\alpha_{1}-\alpha_{2}\right) \sinh Y_{p}+\left(\beta_{1}-\beta_{2}\right) \sinh Y_{q}\right) \\
& \\
&+\left(0, q_{1}^{\perp} \cos \theta_{1}-q_{2}^{\perp} \cos \theta_{2}, q_{1}^{\perp} \sin \theta_{1}-q_{2}^{\perp} \sin \theta_{2}, 0\right) \\
& \longrightarrow\left(\frac{\sqrt{s}}{2}\left(\alpha_{1}+\beta_{1}-\alpha_{2}-\beta_{2}\right), q_{1}^{\perp} \cos \theta_{1}-q_{2}^{\perp} \cos \theta_{2},\right. \\
&\left.q_{1}^{\perp}-q_{2}^{\perp} \sin \theta_{2}, \frac{\sqrt{s}}{2}\left(\alpha_{1}-\beta_{1}-\alpha_{2}+\beta_{2}\right)\right) .
\end{aligned}
$$

The associated energies are read off these expressions to be

$$
\begin{aligned}
& \omega_{p^{\prime}}=\frac{\sqrt{s}}{2}\left(1-\alpha_{1}-\beta_{1}\right), \\
& \omega_{q^{\prime}}=\frac{\sqrt{s}}{2}\left(1+\alpha_{2}+\beta_{2}\right), \\
& \omega_{k}=\frac{\sqrt{s}}{2}\left(\alpha_{1}+\beta_{1}-\alpha_{2}-\beta_{2}\right),
\end{aligned}
$$

which obviously satisfy energy conservation, $\omega_{p^{\prime}}+\omega_{q^{\prime}}+\omega_{k}=\sqrt{s}$. In addition, the on-shell condition for the outgoing momenta fixes the magnitude of the transverse momenta in terms of the Sudakov parameters

$$
\begin{array}{lll}
p^{\prime 2}=0 & \Longrightarrow & \left|Q_{1}\right|^{2}=s\left(\alpha_{1}-1\right) \beta_{1}, \\
q^{\prime 2}=0 & \Longrightarrow & \left|Q_{2}\right|^{2}=s \alpha_{2}\left(1+\beta_{2}\right), \\
k^{2}=0 & \Longrightarrow & \left|Q_{1}-Q_{2}\right|^{2}=s\left(\alpha_{1}-\alpha_{2}\right)\left(\beta_{1}-\beta_{2}\right) .
\end{array}
$$

In fact, combining them we find a further identity

$$
Q_{1} Q_{2}^{*}+Q_{1}^{*} Q_{2}=s\left(\alpha_{2}-\beta_{1}+\alpha_{1} \beta_{2}+\alpha_{2} \beta_{1}\right)
$$

It is important to stress at this point that, unlike the situation encountered in the fourpoint amplitude, here the on-shell conditions for the outgoing particles do not lead to consistency identities restricting the values of the Sudakov parameters. Thus, whereas in the case of four particles the identity (4.13) implies the existence of a single independent Sudakov parameter, in the five-point amplitude the four parameters remain independent.

The coordinates of the punctures associated with each momenta corresponding to Fairlie's solution are given by

$$
\begin{aligned}
\sigma_{p^{\prime}} & =\frac{Q_{1}}{\beta_{1} \sqrt{s}}=\sqrt{\frac{\alpha_{1}-1}{\beta_{1}}} e^{i \theta_{1}}=e^{Y_{p^{\prime}}+i \phi_{p^{\prime}}}, \\
\sigma_{q^{\prime}} & =\frac{Q_{2}}{\left(1+\beta_{2}\right) \sqrt{s}}=\sqrt{\frac{\alpha_{2}}{1+\beta_{2}}} e^{i \theta_{2}}=e^{Y_{q^{\prime}}+i \phi_{q^{\prime}},} \\
\sigma_{k} & =\frac{Q_{1}-Q_{2}}{\left(\beta_{1}-\beta_{2}\right) \sqrt{s}}=\frac{\sqrt{\left(\alpha_{1}-1\right) \beta_{1}} e^{i \theta_{1}}-\sqrt{\left(1+\beta_{2}\right) \alpha_{2}} e^{i \theta_{2}}}{\beta_{1}-\beta_{2}}=e^{Y_{k}+i \phi_{k}},
\end{aligned}
$$


which are the stereographic coordinates labelling the directions of flight of the particles. In order to visualize the position of these punctures, it is convenient to use the unity vectors

$$
\begin{aligned}
\mathbf{u}_{p^{\prime}}=\frac{2}{\sqrt{s}\left(1-\alpha_{1}-\beta_{1}\right)} & \\
& \times\left(-q_{1}^{\perp} \cos \theta_{1},-q_{1}^{\perp} \sin \theta_{1}, \frac{\sqrt{s}}{2}\left(1-\alpha_{1}+\beta_{1}\right)\right) \\
\mathbf{u}_{q^{\prime}}= & \frac{2}{\sqrt{s}\left(1+\alpha_{2}+\beta_{2}\right)} \\
& \times\left(q_{2}^{\perp} \cos \theta_{2}, q_{2}^{\perp} \sin \theta_{2}, \frac{\sqrt{s}}{2}\left(-1+\alpha_{2}-\beta_{2}\right)\right) \\
\mathbf{u}_{k}=\frac{2}{\sqrt{s}\left(\alpha_{1}+\beta_{1}-\alpha_{2}-\beta_{2}\right)} & \\
\quad & \left(q_{1}^{\perp} \cos \theta_{1}-q_{2}^{\perp} \cos \theta_{2}, q_{1}^{\perp} \sin \theta_{1}-q_{2}^{\perp} \sin \theta_{2}, \frac{\sqrt{s}}{2}\left(\alpha_{1}-\beta_{1}-\alpha_{2}+\beta_{2}\right)\right) .
\end{aligned}
$$

Using the expression for $q_{i}^{\perp}$ given in eq. (5.9), we see that the projections of $\mathbf{u}_{p^{\prime}}$ and $\mathbf{u}_{q^{\prime}}$ lie onto the equatorial plane on circumferences with radii

$$
\begin{aligned}
& R_{p^{\prime}}=2 \sqrt{\frac{\left(\alpha_{1}-1\right) \beta_{1}}{\left(1-\alpha_{1}-\beta_{1}\right)^{2}}}, \\
& R_{q^{\prime}}=2 \sqrt{\frac{\alpha_{2}\left(1+\beta_{2}\right)}{\left(1+\alpha_{2}+\beta_{2}\right)^{2}}} .
\end{aligned}
$$

For the momentum $k$, we just need to notice that since $\theta_{1}$ and $\theta_{2}$ are respectively the arguments of $Q_{1}$ and $Q_{2}$

$$
\begin{aligned}
& q_{1}^{\perp} \cos \theta_{1}-q_{2}^{\perp} \cos \theta_{2}=\operatorname{Re}\left(Q_{1}-Q_{2}\right), \\
& q_{1}^{\perp} \sin \theta_{1}-q_{2}^{\perp} \sin \theta_{2}=\operatorname{Im}\left(Q_{1}-Q_{2}\right) .
\end{aligned}
$$

Hence, the equatorial projection of $\mathbf{u}_{k}$ lies on a circumference of radius

$$
R_{k}=\frac{2\left|Q_{1}-Q_{2}\right|}{\alpha_{1}+\beta_{1}-\alpha_{2}-\beta_{2}}=2 \sqrt{\frac{\left(\alpha_{1}-\alpha_{2}\right)\left(\beta_{1}-\beta_{2}\right)}{\left(\alpha_{1}+\beta_{1}-\alpha_{2}-\beta_{2}\right)^{2}}} .
$$

In figure 4 we show a typical configuration for the five punctures on the Riemann sphere. Several factorization channels can be identified in the expressions given in this section. An interesting one corresponds to $\beta_{1}, \alpha_{2} \rightarrow 0$, with both $\alpha_{1}$ and $-\beta_{2}$ not close to 1. This limit sends the puncture associated with $p^{\prime}$ to the north pole, while the puncture for $q^{\prime}$ approaches the south pole. In this limit the puncture for $k$ remains at the equator whenever $\alpha_{1}+\beta_{2}=0$. Alternatively, we can keep $\sigma_{k}$ at the equator by taking $\alpha_{1},-\beta_{2} \rightarrow 1$, with $\beta_{1}$ and $\alpha_{2}$ not close to 0 . On the other hand, the puncture associated with $k$ moves to the south pole in the limit $\alpha_{1} \rightarrow \alpha_{2}$ and to the north pole if $\beta_{1} \rightarrow \beta_{2}$. 


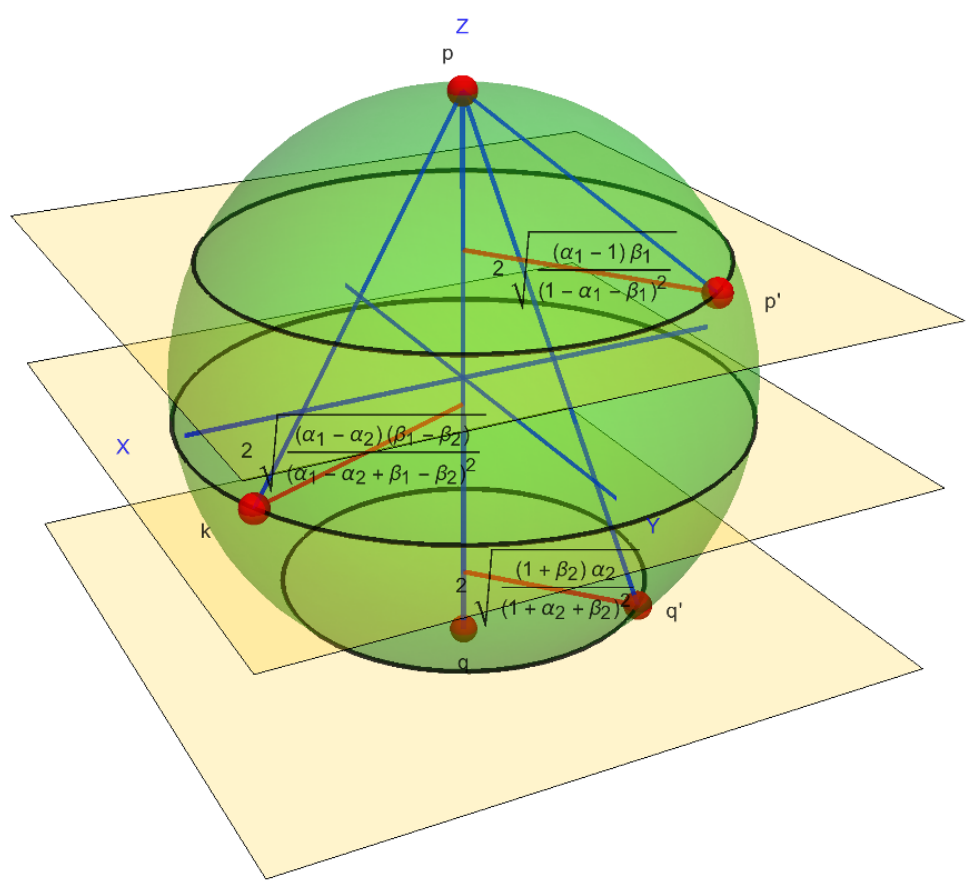

Figure 4. Punctures on the Riemann sphere for the five-particle amplitude.

\subsection{Scattering equations}

To write the SE, we begin by computing the Mandelstam invariants (2.5) in terms of the Sudakov parameters for the five-point amplitude

$$
\begin{aligned}
& s_{p q}=s, \quad s_{p^{\prime} k}=-s\left(\alpha_{2}+\beta_{2}\right), \quad s_{q^{\prime} k}=s\left(\alpha_{1}+\beta_{1}\right), \\
& s_{p p^{\prime}}=-s \beta_{1}, \quad s_{q q^{\prime}}=s \alpha_{2}, \quad s_{p k}=s\left(\beta_{1}-\beta_{2}\right) \text {, } \\
& s_{q k}=s\left(\alpha_{1}-\alpha_{2}\right), \quad s_{p q^{\prime}}=s\left(1+\beta_{2}\right), \quad s_{q p^{\prime}}=s\left(1-\alpha_{1}\right) \text {, } \\
& s_{p^{\prime} q^{\prime}}=s\left(1-\alpha_{1}+\alpha_{2}-\beta_{1}+\beta_{2}\right) \text {. }
\end{aligned}
$$

By inverting these relations, it is possible to express the Sudakov parameters in terms of the invariants as

$$
\begin{array}{ll}
s \alpha_{1}=s_{q^{\prime} k}+s_{p p^{\prime}}, & s \alpha_{2}=s_{q q^{\prime}}, \\
s \beta_{1}=-s_{p p^{\prime}}, & s \beta_{2}=-s_{p^{\prime} k}-s_{q q^{\prime}} .
\end{array}
$$

We know that for the five-point amplitude there must be two different solutions. One of them is the one found by Fairlie [6, 7] that we have expressed in eq. (5.13) in terms of Sudakov parameters. To find the second one, we write the ansatz

$$
\begin{aligned}
& \sigma_{p^{\prime}}=C_{p} \widehat{Q}_{1}, \\
& \sigma_{q^{\prime}}=C_{q} \widehat{Q}_{2},
\end{aligned}
$$

with $C_{p}$ and $C_{q}$ two complex constants and we use the rescaled quantities defined in eq. (4.26). A first condition comes from complying with the SE associated to $q$,

$$
\mathcal{S}_{q} \equiv \frac{1-\alpha_{1}}{\sigma_{p^{\prime}}}+\frac{\alpha_{2}}{\sigma_{q^{\prime}}}+\frac{\alpha_{1}-\alpha_{2}}{\sigma_{k}}=0,
$$


which determines $\sigma_{k}$ to be

$$
\sigma_{k}=\left(\alpha_{2}-\alpha_{1}\right)\left(\frac{1-\alpha_{1}}{C_{p} \widehat{Q}_{1}}+\frac{\alpha_{2}}{C_{q} \widehat{Q}_{2}}\right)^{-1} .
$$

Now we impose the $\mathrm{SE}$ associated to $q^{\prime}$, which reads

$$
\mathcal{S}_{q^{\prime}} \equiv \frac{\alpha_{2}}{\sigma_{q^{\prime}}}+\frac{1-\alpha_{1}+\alpha_{2}-\beta_{1}+\beta_{2}}{\sigma_{p^{\prime} q^{\prime}}}+\frac{\alpha_{1}+\beta_{1}}{\sigma_{k q^{\prime}}}=0
$$

leading to the relation

$$
\frac{\sigma_{k}}{\sigma_{q^{\prime}}}=\frac{\left(\alpha_{2}-\alpha_{1}\right) \sigma_{p^{\prime}}}{\alpha_{2} \sigma_{p^{\prime}}+\left(1-\alpha_{1}\right) \sigma_{q^{\prime}}}=\frac{\left(\alpha_{2}-\alpha_{1}-\beta_{1}\right) \sigma_{p^{\prime}}+\left(1+\beta_{2}\right) \sigma_{q^{\prime}}}{\alpha_{2} \sigma_{p^{\prime}}+\left(1-\alpha_{1}-\beta_{1}+\beta_{2}\right) \sigma_{q^{\prime}}}
$$

Using the on-shell relations (5.9), this equation can be equivalently written as

$$
\alpha_{2} \beta_{1} \sigma_{p^{\prime}}^{2}-\left(\widehat{Q}_{1} \widehat{Q}_{2}^{*}+\widehat{Q}_{1}^{*} \widehat{Q}_{2}\right) \sigma_{p^{\prime}} \sigma_{q^{\prime}}+\frac{\left|\widehat{Q}_{1}\right|^{2}\left|\widehat{Q}_{2}\right|^{2}}{\alpha_{2} \beta_{1}} \sigma_{q^{\prime}}^{2}=0 .
$$

Assuming $\sigma_{q^{\prime}} \neq 0$, this is a quadratic equation for the ratio $\frac{\sigma_{p^{\prime}}}{\sigma_{q^{\prime}}}$ whose coefficients are expressed only in terms of the Sudakov parameters. Its two solutions are given by

$$
\frac{\sigma_{p^{\prime}}^{( \pm)}}{\sigma_{q^{\prime}}^{( \pm)}}=\frac{1}{2 \alpha_{2} \beta_{1}}\left[\widehat{Q}_{1} \widehat{Q}_{2}^{*}+\widehat{Q}_{1}^{*} \widehat{Q}_{2} \pm \sqrt{\left(\widehat{Q}_{1} \widehat{Q}_{2}^{*}+\widehat{Q}_{1}^{*} \widehat{Q}_{2}\right)^{2}-4\left|\widehat{Q}_{1}\right|^{2}\left|\widehat{Q}_{2}\right|^{2}}\right]
$$

which admits the simpler form

$$
\begin{aligned}
& \frac{\sigma_{p^{\prime}}^{(+)}}{\sigma_{q^{\prime}}^{(+)}}=\frac{\widehat{Q}_{1} \widehat{Q}_{2}^{*}}{\alpha_{2} \beta_{1}}, \\
& \frac{\sigma_{p^{\prime}}^{(-)}}{\sigma_{q^{\prime}}^{(-)}}=\frac{\widehat{Q}_{1}^{*} \widehat{Q}_{2}}{\alpha_{2} \beta_{1}} .
\end{aligned}
$$

Being solutions to a quadratic equation with real coefficients, they are complex conjugate of each other. Using now the second equation in (5.21), together with (5.25) and the on-shell conditions (5.9), we arrive at the following expression of the solution $\sigma_{i}^{(+)}$to the SE

$$
\begin{aligned}
\sigma_{p^{\prime}}^{(+)} & =C_{q} \frac{\left(1+\beta_{2}\right)}{\beta_{1}} \widehat{Q}_{1}, \\
\sigma_{q^{\prime}}^{(+)} & =C_{q} \widehat{Q}_{2}, \\
\sigma_{k}^{(+)} & =C_{q} \frac{\left(1+\beta_{2}\right)}{\beta_{1}-\beta_{2}}\left(\widehat{Q}_{1}-\widehat{Q}_{2}\right) .
\end{aligned}
$$

To fix the undetermined constant $C_{q}$ we identify $\sigma_{i}^{(+)}$with Fairlie's solution (5.13). This fixes $C_{q}$ to be

$$
C_{q}=\frac{e^{-i \theta_{2}}}{1+\beta_{2}}
$$


In order to understand the presence of the phase in this expression, we should point out that, in setting $\sigma_{p}=\infty$ and $\sigma_{q}=0$, we only partially fixed the $\operatorname{SL}(2, \mathbb{C})$ invariance of the moduli space of punctured spheres. This leaves us with complex rescalings as the residual invariance. We can make use of this freedom to set the phase of the constant $C_{q}$ as in (5.30), which geometrically corresponds to a change in the origin of the azimuthal angles in the Riemann sphere. Our choice, which sets $\sigma_{q^{\prime}}^{( \pm)}$on the real axis, leads to a more symmetric form of the two solutions to the SE for the five-point amplitude

$$
\begin{aligned}
& \sigma_{p^{\prime}}^{(+)}=\sigma_{p^{\prime}}^{(-) *}=\frac{\widehat{Q}_{1} e^{-i \theta_{2}}}{\beta_{1}}=\sqrt{\frac{\alpha_{1}-1}{\beta_{1}}} e^{i\left(\theta_{1}-\theta_{2}+\pi\right)}, \\
& \sigma_{q^{\prime}}^{(+)}=\sigma_{q^{\prime}}^{(-) *}=\frac{\widehat{Q}_{2} e^{-i \theta_{2}}}{1+\beta_{2}}=\sqrt{\frac{\alpha_{2}}{1+\beta_{2}}}, \\
& \sigma_{k}^{(+)}=\sigma_{k}^{(-) *}=\frac{\left(\widehat{Q}_{1}-\widehat{Q}_{2}\right) e^{-i \theta_{2}}}{\beta_{1}-\beta_{2}}=\frac{\sqrt{\left(\alpha_{1}-1\right) \beta_{1}} e^{i\left(\theta_{1}-\theta_{2}\right)}-\sqrt{\alpha_{2}\left(1+\beta_{2}\right)}}{\beta_{1}-\beta_{2}} .
\end{aligned}
$$

The localization of the punctures on the Riemann sphere can be also given in terms of the unit vectors

$$
\begin{aligned}
& \mathbf{u}_{p^{\prime}}^{( \pm)}= \frac{1}{1-\alpha_{1}-\beta_{1}}\left(-2 \sqrt{\left(\alpha_{1}-1\right) \beta_{1}} \cos \gamma, \mp 2 \sqrt{\left(\alpha_{1}-1\right) \beta_{1}} \sin \gamma, 1-\alpha_{1}+\beta_{1}\right), \\
& \mathbf{u}_{q^{\prime}}^{( \pm)}= \frac{1}{\alpha_{2}+\beta_{2}+1}\left(2 \sqrt{\alpha_{2}\left(1+\beta_{2}\right)}, 0, \alpha_{2}-\beta_{2}-1\right), \\
& \mathbf{u}_{k}^{( \pm)}= \frac{1}{\alpha_{1}+\beta_{1}-\alpha_{2}-\beta_{2}}\left(2 \sqrt{\left(\alpha_{1}-1\right) \beta_{1}} \cos \gamma-\sqrt{\alpha_{2}\left(1+\beta_{2}\right)},\right. \\
&\left.\mp 2 \sqrt{\left(\alpha_{1}-1\right) \beta_{1}} \sin \gamma, \alpha_{1}-\beta_{1}-\alpha_{2}+\beta_{2}\right),
\end{aligned}
$$

where we have defined $\gamma=\theta_{1}-\theta_{2}$. As announced, $\sigma_{i}^{(+)}$corresponds to Fairlie's solution, after choosing the origin of azimuthal angles such that $\theta_{2}=0$ in eq. (5.14). The second solution $\sigma_{i}^{(-)}$is obtained by reflecting the first one with respect to the $y=0$ plane.

\subsection{Scalar scattering amplitude}

Having obtained the two solutions to the SE, we are now ready to calculate the five-point amplitude for the $\varphi^{3}$ scalar theory. Using the same partial fixing of $\operatorname{SL}(2, \mathbb{C})$ as in the calculation of the four-point amplitude in eq. (4.32), we are left with the computation of the integral over the position of the punctures associated with $p^{\prime}$ and $q^{\prime}$, namely

$$
\begin{aligned}
\mathcal{A}_{5}^{\varphi^{3}} & =\int d z_{p^{\prime}} d z_{q^{\prime}} \delta\left(\mathcal{S}_{p^{\prime}}\right) \delta\left(\mathcal{S}_{q^{\prime}}\right) \frac{z_{p q}^{2} z_{q k}^{2} z_{k p}^{2}}{\left(z_{p q} z_{q q^{\prime}} z_{q^{\prime} k} z_{k p^{\prime}} z_{p^{\prime} p}\right)^{2}} \\
& =\int d z_{p^{\prime}} d z_{q^{\prime}} \delta\left(\mathcal{S}_{p^{\prime}}\right) \delta\left(\mathcal{S}_{q^{\prime}}\right) \frac{z_{k}^{2}}{z_{q^{\prime}}^{2} z_{q^{\prime} k}^{2} z_{k p^{\prime}}^{2}} .
\end{aligned}
$$

To solve the delta function, we have to calculate the Jacobian

$$
\mathcal{J}=\frac{\partial \mathcal{S}_{p^{\prime}}}{\partial \sigma_{p^{\prime}}} \frac{\partial \mathcal{S}_{q^{\prime}}}{\partial \sigma_{q^{\prime}}}-\frac{\partial \mathcal{S}_{p^{\prime}}}{\partial \sigma_{q^{\prime}}} \frac{\partial \mathcal{S}_{q^{\prime}}}{\partial \sigma_{p^{\prime}}} .
$$


Things can be made simpler if we rewrite the SE associated to $q^{\prime}$ in the form

$$
\begin{aligned}
\frac{1}{s} \mathcal{S}_{q^{\prime}} & =\frac{\alpha_{2}}{\sigma_{q^{\prime}}}+\frac{\left(1-\alpha_{1}+\alpha_{2}-\beta_{1}+\beta_{2}\right)}{\sigma_{p^{\prime} q^{\prime}}}+\frac{\left(\alpha_{1}+\beta_{1}\right)}{\sigma_{k q^{\prime}}} \\
& =\frac{\left(\alpha_{2} \sigma_{p^{\prime}}+\left(1-\alpha_{1}-\beta_{1}+\beta_{2}\right) \sigma_{q^{\prime}}\right)}{\sigma_{p^{\prime} q^{\prime}} \sigma_{k q^{\prime}}}\left[\frac{\sigma_{k}}{\sigma_{q^{\prime}}}-\frac{\left(\alpha_{2}-\alpha_{1}-\beta_{1}\right) \sigma_{p^{\prime}}+\left(1+\beta_{2}\right) \sigma_{q^{\prime}}}{\alpha_{2} \sigma_{p^{\prime}}+\left(1-\alpha_{1}-\beta_{1}+\beta_{2}\right) \sigma_{q^{\prime}}}\right] .
\end{aligned}
$$

What makes this expression useful is that we have isolated the zero due to eq. (5.25). We can then write one of the derivatives on support on the SE as

$$
\begin{aligned}
\left.\frac{1}{s} \frac{\partial \mathcal{S}_{q^{\prime}}}{\partial \sigma_{q^{\prime}}}\right|_{\mathrm{SE}} & =\frac{\alpha_{2} \sigma_{p^{\prime}}+\left(1-\alpha_{1}-\beta_{1}+\beta_{2}\right) \sigma_{q^{\prime}}}{\sigma_{p^{\prime} q^{\prime}} \sigma_{k q^{\prime}}} \frac{\partial}{\partial \sigma_{q^{\prime}}}\left[\frac{\sigma_{k}}{\sigma_{q^{\prime}}}-\frac{\left(\alpha_{2}-\alpha_{1}-\beta_{1}\right) \sigma_{p^{\prime}}+\left(1+\beta_{2}\right) \sigma_{q^{\prime}}}{\alpha_{2} \sigma_{p^{\prime}}+\left(1-\alpha_{1}-\beta_{1}+\beta_{2}\right) \sigma_{q^{\prime}}}\right] \\
& =\frac{\left(\alpha_{1}-\alpha_{2}-\beta_{1}\right) \alpha_{2} \sigma_{p^{\prime}}+\left[\left(\alpha_{1}-\alpha_{2}\right) \beta_{2}+\left(\alpha_{2}-1\right) \beta_{1}\right] \sigma_{q^{\prime}}}{\alpha_{2} \sigma_{p^{\prime}}+\left(1-\alpha_{1}\right) \sigma_{q^{\prime}}}\left(\frac{\sigma_{p^{\prime}}}{\sigma_{q^{\prime}} \sigma_{p^{\prime} q^{\prime}} \sigma_{k q^{\prime}}}\right),
\end{aligned}
$$

where we have used eqs. (5.25) and (5.26). This can be further simplified by reintroducing $\sigma_{k}$ to write

$$
\left.\frac{\partial \mathcal{S}_{q^{\prime}}}{\partial \sigma_{q^{\prime}}}\right|_{\mathrm{SE}}=s \frac{\left(1+\beta_{2}\right) \sigma_{q^{\prime}}^{2}+\alpha_{2} \sigma_{k} \sigma_{p^{\prime}}}{\sigma_{q^{\prime}}^{2} \sigma_{p^{\prime} q^{\prime}} \sigma_{q^{\prime} k}} .
$$

We now repeat the same procedure for the SE associated to $p^{\prime}$, isolating the contribution to the zero

$$
\frac{1}{s} \mathcal{S}_{p^{\prime}}=\frac{\left(\alpha_{2}-\beta_{1}+\beta_{2}\right) \sigma_{p^{\prime}}+\left(1-\alpha_{1}\right) \sigma_{q^{\prime}}}{\sigma_{p^{\prime} q^{\prime}} \sigma_{p^{\prime} k}}\left[\frac{\sigma_{k}}{\sigma_{p^{\prime}}}+\frac{\left(\alpha_{1}-1-\alpha_{2}-\beta_{2}\right) \sigma_{q^{\prime}}+\beta_{1} \sigma_{p^{\prime}}}{\left(\alpha_{2}-\beta_{1}+\beta_{2}\right) \sigma_{p^{\prime}}+\left(1-\alpha_{1}\right) \sigma_{q^{\prime}}}\right],
$$

and differentiating with respect to $\sigma_{p^{\prime}}$, we find

$$
\left.\frac{\partial \mathcal{S}_{p^{\prime}}}{\partial \sigma_{p^{\prime}}}\right|_{\mathrm{SE}}=s \frac{\beta_{1} \sigma_{p^{\prime}}^{2}+\left(\alpha_{1}-1\right) \sigma_{k} \sigma_{q^{\prime}}}{\sigma_{p^{\prime}}^{2} \sigma_{p^{\prime} q^{\prime}} \sigma_{p^{\prime} k}} .
$$

Note that the two partial derivatives (5.39) and (5.41) can be mapped to each other by the replacements

$$
\begin{aligned}
p^{\prime} & \longleftrightarrow q^{\prime}, \\
-\beta_{1} & \longleftrightarrow 1+\beta_{2}, \\
\alpha_{1} & \longleftrightarrow 1-\alpha_{2} .
\end{aligned}
$$

Similar techniques allow to obtain the remaining two derivatives

$$
\begin{aligned}
& \left.\frac{\partial \mathcal{S}_{q^{\prime}}}{\partial \sigma_{p^{\prime}}}\right|_{\mathrm{SE}}=s \frac{\left(\alpha_{1}+\beta_{1}\right)\left(1-\alpha_{1}+\alpha_{2}-\beta_{1}+\beta_{2}\right)}{\left(\beta_{1}-\beta_{2}\right) \sigma_{k}+\left(\alpha_{1}-\alpha_{2}\right) \sigma_{p^{\prime}}}\left(\frac{\sigma_{k}}{\sigma_{p^{\prime} q^{\prime}} \sigma_{q^{\prime} k}}\right), \\
& \left.\frac{\partial \mathcal{S}_{p^{\prime}}}{\partial \sigma_{q^{\prime}}}\right|_{\mathrm{SE}}=s \frac{\left(1-\alpha_{1}+\alpha_{2}-\beta_{1}+\beta_{2}\right)\left(\alpha_{2}+\beta_{2}\right)}{\left(\beta_{1}-\beta_{2}\right) \sigma_{k}+\left(\alpha_{1}-\alpha_{2}\right) \sigma_{q^{\prime}}}\left(\frac{\sigma_{k}}{\sigma_{p^{\prime} q^{\prime}} \sigma_{p^{\prime} k}}\right),
\end{aligned}
$$

which are also related by the transformations (5.42). 
We can return to the calculation of the amplitude, which can be written as the sum over the two solutions complex conjugate of each other:

$$
\begin{aligned}
\mathcal{A}_{5}^{\varphi^{3}} & =\int d z_{p^{\prime}} d z_{q^{\prime}} \mathcal{J}^{-1} \delta\left(z_{p^{\prime}}-\sigma_{p^{\prime}}\right) \delta\left(z_{q^{\prime}}-\sigma_{q^{\prime}}\right) \frac{z_{k}^{2}}{z_{q^{\prime}}^{2} z_{q^{\prime} k}^{2} z_{k p^{\prime}}^{2}}+\text { c.c. } \\
& =\frac{2}{s^{2}} \operatorname{Re}\left[\left(\frac{\sigma_{p^{\prime} q^{\prime}}^{2}}{\sigma_{q^{\prime} k} \sigma_{p^{\prime} k}}\right) \frac{1}{\mathcal{L}-\mathcal{R}}\right] \\
& =\frac{1}{s^{2}}\left[\frac{1}{\alpha_{1}+\beta_{1}}-\frac{1}{\alpha_{2}+\beta_{2}}+\frac{1}{\left(\alpha_{1}+\beta_{1}\right) \beta_{1}}-\frac{1}{\beta_{1} \alpha_{2}}+\frac{1}{\alpha_{2}\left(\alpha_{2}+\beta_{2}\right)}\right] .
\end{aligned}
$$

Here we have used the notation

$$
\begin{aligned}
\mathcal{L} & \equiv\left[\beta_{1} \frac{\sigma_{p^{\prime}}}{\sigma_{k}}+\left(\alpha_{1}-1\right) \frac{\sigma_{q^{\prime}}}{\sigma_{p^{\prime}}}\right]\left[\alpha_{2}+\left(1+\beta_{2}\right) \frac{\sigma_{q^{\prime}}^{2}}{\sigma_{k} \sigma_{p^{\prime}}}\right], \\
\mathcal{R} & \equiv \frac{\left(1-\alpha_{1}+\alpha_{2}-\beta_{1}+\beta_{2}\right)^{2}\left(\alpha_{1}+\beta_{1}\right)\left(\alpha_{2}+\beta_{2}\right) \sigma_{q^{\prime}}^{2}}{\left[\left(\beta_{1}-\beta_{2}\right) \sigma_{k}+\left(\alpha_{1}-\alpha_{2}\right) \sigma_{p^{\prime}}\right]\left[\left(\beta_{1}-\beta_{2}\right) \sigma_{k}+\left(\alpha_{1}-\alpha_{2}\right) \sigma_{q^{\prime}}\right]},
\end{aligned}
$$

as well as the explicit expressions for the positions of the punctures given in eq. (5.31). In fact, the on-shell conditions (5.9) can be written in the form

$$
2 \cos \left(\theta_{1}-\theta_{2}\right)=\frac{\alpha_{2}-\beta_{1}+\alpha_{1} \beta_{2}+\alpha_{2} \beta_{1}}{\sqrt{\left(\alpha_{1}-1\right) \beta_{1}} \sqrt{\left(1+\beta_{2}\right) \alpha_{2}}},
$$

which is useful for the numerical evaluation of our expressions and shows how the relative phase depends on the Sudakov variables.

In fact, it is possible to write an alternative expression for the amplitude (5.44) as

$$
\mathcal{A}_{5}^{\varphi^{3}}=\frac{2}{s^{2}} \operatorname{Re}\left[\left(\frac{\sigma_{p^{\prime}}}{\sigma_{q^{\prime}}}\right) \frac{1}{L \widetilde{L}-R \widetilde{R}}\right]
$$

where

$$
\begin{aligned}
& L=\frac{\sigma_{p^{\prime} k}}{\sigma_{p^{\prime} q^{\prime}}}\left[\left(\alpha_{1}-1\right) \frac{\sigma_{q^{\prime}}}{\sigma_{p^{\prime}}}+\beta_{1} \frac{\sigma_{p^{\prime}}}{\sigma_{q^{\prime}}}\right], \\
& R=\left(\frac{\sigma_{p^{\prime}} \sigma_{p^{\prime} k}}{\sigma_{p^{\prime} q^{\prime}}}\right) \frac{\left(1-\alpha_{1}+\alpha_{2}-\beta_{1}+\beta_{2}\right)\left(\alpha_{1}+\beta_{1}\right)}{\left(\alpha_{1}-\alpha_{2}+\beta_{1}\right) \sigma_{p^{\prime}}-\left(1+\beta_{2}\right) \sigma_{q^{\prime}}},
\end{aligned}
$$

and the quantities with tilde are defined by implementing the replacements (5.42) in the form

$$
\widetilde{\mathscr{O}}\left(\alpha_{1}, \alpha_{2}, \beta_{1}, \beta_{2}, \theta_{1}-\theta_{2}\right)=\mathscr{O}\left(1-\alpha_{2}, 1-\alpha_{1},-1-\beta_{2},-1-\beta_{1}, \theta_{2}-\theta_{1}\right) .
$$

The reason behind the existence of such a simple representation is the freedom to redefine the phase in the projective variables. This is part of the residual $\operatorname{SL}(2, \mathbb{C})$ freedom present in our approach, after fixing the punctures associated with the two incoming particles. One interesting feature of eq. (5.48) is related to the mathematical properties of the zeros of this amplitude, an issue which has already been explored in [38, 39]. This will be further investigated in a future publication. 


\section{Conclusions and outlook}

We have presented a first complete analysis on the use of Sudakov variables in the context of the CHY calculation of scattering amplitudes. These amplitudes are represented as integrals with support on the solution to the scattering equations. Using these variables and a particular frame for the two incoming particles, it is possible to clearly identify the solutions to the scattering equations as punctures on the Riemann sphere parametrized by the rapidity and azimuthal angle, defined on the transverse plane to the collision axis of the incoming particles, of each on-shell particle. The punctures for the emitted particles are then living on circles parametrized by one Sudakov variable, in the four-point case, and four Sudakov variables for five-particles amplitude. In this formulation of the CHY calculation the final expression for a scalar amplitude has a very simple structure, given in terms of the position of the punctures which are complex numbers carrying a phase defined as the difference of azimuthal angles of the emitted particles.

In a future work we will generalize the Sudakov representation for a $n$-point amplitude and show the different multi-particle factorization limits which are naturally parametrized in this approach. The connection among gravitational and Yang-Mills amplitudes in this approach from the point of view of Regge kinematics [40-43] is also of interest, together with the corresponding soft theorems [35, 44-48]. Besides, it would be interesting to interpret the role of the gluing operator recently investigated in [37] in terms of Sudakov variables. Certainly, the relevance of this operator for the calculation of higher-loop amplitudes is still to be investigated and exploring kinematical limits, such as multi-Regge kinematics where the Sudakov representation is most useful, could be a possible route to understand its meaning.

\section{Acknowledgments}

We would like to thank David Skinner, Piotr Tourkine and Ellis Y. Yuan for very useful discussions. A.S.V. would like to thank David Skinner for the warm hospitality at the Department of Applied Mathematics and Theoretical Physics (DAMTP), at the University of Cambridge, during the summer of 2017. G.C., A.S.V. and D.M.J. acknowledge support from the Spanish Government grants FPA2015-65480-P, FPA2016-78022-P and Spanish MINECO Centro de Excelencia Severo Ochoa Programme (SEV-2016-0597). M.A.V.-M. has been partially supported by Spanish Government grant FPA2015-64041-C2-2-P and gratefully acknowledges the hospitality of the KEK Theory Center during the completion of this work.

Open Access. This article is distributed under the terms of the Creative Commons Attribution License (CC-BY 4.0), which permits any use, distribution and reproduction in any medium, provided the original author(s) and source are credited.

\section{References}

[1] H. Elvang and Y.T. Huang, Scattering amplitudes in gauge theory and gravity, Cambridge Unviersity Press, Cambridge U.K. (2015). 
[2] F. Cachazo, S. He and E.Y. Yuan, Scattering of massless particles in arbitrary dimensions, Phys. Rev. Lett. 113 (2014) 171601 [arXiv:1307.2199] [InSPIRE].

[3] F. Cachazo, S. He and E.Y. Yuan, Scattering of massless particles: scalars, gluons and gravitons, JHEP 07 (2014) 033 [arXiv: 1309.0885] [INSPIRE].

[4] D.J. Gross and P.F. Mende, The high-energy behavior of string scattering amplitudes, Phys. Lett. B 197 (1987) 129 [inSPIRE].

[5] D.J. Gross and P.F. Mende, String theory beyond the Planck scale, Nucl. Phys. B 303 (1988) 407 [INSPIRE].

[6] D.B. Fairlie, A coding of real null four-momenta into world-sheet coordinates, Adv. Math. Phys. 2009 (2009) 284689 [arXiv: 0805. 2263] [INSPIRE].

[7] D.B. Fairlie and D.E. Roberts, Dual models without tachyons - A new approach, Durham preprint PRINT-72-2440 (1972).

[8] L. Dolan and P. Goddard, Proof of the formula of cachazo, He and Yuan for Yang-Mills tree amplitudes in arbitrary dimension, JHEP 05 (2014) 010 [arXiv: 1311.5200] [INSPIRE].

[9] L. Mason and D. Skinner, Ambitwistor strings and the scattering equations, JHEP 07 (2014) 048 [arXiv: 1311.2564] [INSPIRE].

[10] N. Berkovits, Infinite tension limit of the pure spinor superstring, JHEP 03 (2014) 017 [arXiv: 1311.4156] [INSPIRE].

[11] T. Adamo, E. Casali and D. Skinner, Ambitwistor strings and the scattering equations at one loop, JHEP 04 (2014) 104 [arXiv: 1312.3828] [INSPIRE].

[12] K. Ohmori, Worldsheet geometries of ambitwistor string, JHEP 06 (2015) 075 [arXiv: 1504.02675] [INSPIRE].

[13] E. Casali et al., New ambitwistor string theories, JHEP 11 (2015) 038 [arXiv:1506.08771] [INSPIRE].

[14] T. Adamo and E. Casali, Scattering equations, supergravity integrands and pure spinors, JHEP 05 (2015) 120 [arXiv: 1502.06826] [INSPIRE].

[15] Y. Geyer, L. Mason, R. Monteiro and P. Tourkine, Loop integrands for scattering amplitudes from the Riemann sphere, Phys. Rev. Lett. 115 (2015) 121603 [arXiv:1507.00321] [INSPIRE].

[16] Y. Geyer, L. Mason, R. Monteiro and P. Tourkine, One-loop amplitudes on the Riemann sphere, JHEP 03 (2016) 114 [arXiv:1511.06315] [INSPIRE].

[17] N.E.J. Bjerrum-Bohr, P.H. Damgaard, P. Tourkine and P. Vanhove, Scattering equations and string theory amplitudes, Phys. Rev. D 90 (2014) 106002 [arXiv:1403.4553] [INSPIRE].

[18] H. Gomez and E.Y. Yuan, $N$-point tree-level scattering amplitude in the new Berkovits' string, JHEP 04 (2014) 046 [arXiv: 1312.5485] [INSPIRE].

[19] E. Casali and P. Tourkine, Infrared behaviour of the one-loop scattering equations and supergravity integrands, JHEP 04 (2015) 013 [arXiv: 1412.3787] [INSPIRE].

[20] S. Weinzierl, On the solutions of the scattering equations, JHEP 04 (2014) 092 [arXiv: 1402.2516] [INSPIRE].

[21] L. Dolan and P. Goddard, The polynomial form of the scattering equations, JHEP 07 (2014) 029 [arXiv: 1402.7374] [INSPIRE]. 
[22] C.S. Lam, Permutation symmetry of the scattering equations, Phys. Rev. D 91 (2015) 045019 [arXiv: 1410.8184] [INSPIRE].

[23] C. Kalousios, Scattering equations, generating functions and all massless five point tree amplitudes, JHEP 05 (2015) 054 [arXiv: 1502.07711] [INSPIRE].

[24] C. Baadsgaard, N.E.J. Bjerrum-Bohr, J.L. Bourjaily and P.H. Damgaard, Integration rules for scattering equations, JHEP 09 (2015) 129 [arXiv: 1506.06137] [INSPIRE].

[25] C. Baadsgaard, N.E.J. Bjerrum-Bohr, J.L. Bourjaily and P.H. Damgaard, Scattering equations and Feynman diagrams, JHEP 09 (2015) 136 [arXiv:1507.00997] [INSPIRE].

[26] C. Cardona and C. Kalousios, Elimination and recursions in the scattering equations, Phys. Lett. B 756 (2016) 180 [arXiv:1511.05915] [INSPIRE].

[27] L. Dolan and P. Goddard, General solution of the scattering equations, JHEP 10 (2016) 149 [arXiv: 1511.09441] [INSPIRE].

[28] V.V. Sudakov, Vertex parts at very high-energies in quantum electrodynamics, Sov. Phys. JETP 3 (1956) 65 [Zh. Eksp. Teor. Fiz. 30 (1956) 87] [INSPIRE].

[29] D.J. Gross and J.L. Mañes, The high-energy behavior of open string scattering, Nucl. Phys. B 326 (1989) 73 [INSPIRE].

[30] H. Kawai, D.C. Lewellen and S.H.H. Tye, A relation between tree amplitudes of closed and open strings, Nucl. Phys. B 269 (1986) 1 [INSPIRE].

[31] Z. Bern, J.J.M. Carrasco and H. Johansson, New relations for gauge-theory amplitudes, Phys. Rev. D 78 (2008) 085011 [arXiv: 0805.3993] [INSPIRE].

[32] Z. Bern, J.J.M. Carrasco and H. Johansson, Perturbative quantum gravity as a double copy of gauge theory, Phys. Rev. Lett. 105 (2010) 061602 [arXiv: 1004.0476] [INSPIRE].

[33] R. Monteiro and D. O'Connell, The kinematic algebras from the scattering equations, JHEP 03 (2014) 110 [arXiv:1311.1151] [INSPIRE].

[34] S.G. Naculich, Scattering equations and BCJ relations for gauge and gravitational amplitudes with massive scalar particles, JHEP 09 (2014) 029 [arXiv:1407.7836] [INSPIRE].

[35] A. Sabio Vera and M.Á. Vázquez-Mozo, The double copy structure of soft gravitons, JHEP 03 (2015) 070 [arXiv: 1412.3699] [inSPIRE].

[36] R. Monteiro, D. O'Connell and C.D. White, Black holes and the double copy, JHEP 12 (2014) 056 [arXiv: 1410.0239] [INSPIRE].

[37] K.A. Roehrig and D. Skinner, A gluing operator for the ambitwistor string, arXiv: 1709.03262 [INSPIRE].

[38] D. Medrano Jiménez, A. Sabio Vera and M.Á. Vázquez-Mozo, Planar zeros in gauge theories and gravity, JHEP 09 (2016) 006 [arXiv: 1607.04605] [INSPIRE].

[39] D. Medrano Jiménez, A. Sabio Vera and M.Á. Vázquez-Mozo, Projectivity of planar zeros in field and string theory amplitudes, JHEP 05 (2017) 011 [arXiv: 1703.07274] [INSPIRE].

[40] A. Sabio Vera, E. Serna Campillo and M.Á. Vázquez-Mozo, Graviton emission in Einstein-Hilbert gravity, JHEP 03 (2012) 005 [arXiv:1112.4494] [INSPIRE].

[41] A. Sabio Vera, E. Serna Campillo and M.Á. Vázquez-Mozo, Color-kinematics duality and the Regge limit of inelastic amplitudes, JHEP 04 (2013) 086 [arXiv:1212.5103] [INSPIRE]. 
[42] H. Johansson, A. Sabio Vera, E. Serna Campillo and M. Á. Vázquez-Mozo, Color-kinematics duality in multi-Regge kinematics and dimensional reduction, JHEP 10 (2013) 215 [arXiv: 1307.3106] [INSPIRE].

[43] H. Johansson, A. Sabio Vera, E. Serna Campillo and M.Á. Vázquez-Mozo, Color-kinematics duality and dimensional reduction for graviton emission in Regge limit, arXiv:1310.1680 [INSPIRE].

[44] B.U.W. Schwab and A. Volovich, Subleading soft theorem in arbitrary dimensions from scattering equations, Phys. Rev. Lett. 113 (2014) 101601 [arXiv:1404.7749] [INSPIRE].

[45] S. He, Y.-t. Huang and C. Wen, Loop corrections to soft theorems in gauge theories and gravity, JHEP 12 (2014) 115 [arXiv: 1405.1410] [INSPIRE].

[46] M. Bianchi, S. He, Y.-t. Huang and C. Wen, More on soft theorems: trees, loops and strings, Phys. Rev. D 92 (2015) 065022 [arXiv:1406.5155] [INSPIRE].

[47] F. Cachazo, S. He and E.Y. Yuan, New double soft emission theorems, Phys. Rev. D 92 (2015) 065030 [arXiv: 1503.04816] [InSPIRE].

[48] P. Di Vecchia, R. Marotta and M. Mojaza, Double-soft behavior for scalars and gluons from string theory, JHEP 12 (2015) 150 [arXiv: 1507.00938] [INSPIRE]. 